\title{
Mean first passage time and the Kramers turnover theory in activated atom-surface diffusion $\dagger$
}

\author{
J. L. Vega, R. Guantes and S. Miret-Artés
}

Instituto de Matemáticas y Física Fundamental, Consejo Superior de Investigaciones Científicas, Serrano 123,28006, Madrid, Spain.E-mail: salvador@fam10.imaff.csic.es

\author{
Received 22nd May 2002, Accepted 9th August 2002 \\ First published as an Advance Article on the web 5th September 2002
}

\begin{abstract}
Calculations of noise-assisted jump rates and diffusion coefficients for diffusion of atoms adsorbed on a metal surface are presented and discussed, in the whole range of the damping strength, and with a direct numerical integration of the Langevin equation, by two different procedures: a mean first passage time calculation and by counting jumps with a given energy criterion. The results are compared to the analytical ones by using the extension of the Kramers model by Mel'nikov and Meshkov. The role of the adiabatic potential coupling in the jump rates, jump distributions and diffusion coefficients is analysed and results are finally compared to previous experimental findings for the $\mathrm{Na} / \mathrm{Cu}(001)$ system.
\end{abstract}

\section{Introduction}

In transport phenomena, one of the basic concepts in activated processes is the total jump rate from going from one stationary state to another by crossing a potential barrier, located in between, together with the corresponding jump mechanism governing a given physical problem, considered as a stochastic process. It has been quite a tradition, in the past, to compare experimentally measured jump rates with those predicted by the 'standard' transition state theory (TST) ${ }^{1}$ and it is still commonly taken as the reference starting point for discussion of gas phase and condensed phase chemical reactions ${ }^{1-3}$ and surface diffusion processes. ${ }^{4,5}$ Inspired by a work by Christiansen, ${ }^{6}$ where a chemical reaction was considered as a diffusion problem, Kramers ${ }^{7}$ introduced a Brownian motion model in a onedimensional (along the reaction coordinate) force field to predict the existence of several kinetic regimes depending on the magnitude of the friction (very low or energy diffusion regime, and moderate to high or spatial diffusion regime). A generalization to the TST results and an extension to deal with damping processes were finally reached. From then, many authors have devised analytical methods for obtaining escape rates in the whole range of friction by extending the basic assumptions found in the original Kramers work and known as the Kramers turnover problem (see for example the reviews ${ }^{1-3,8,9}$ ).

The Kramers equation, a Fokker-Planck type equation, is quite complicated to solve and analytical solutions are only possible for very simple interaction models. ${ }^{10}$ A numerical solution for one and two-dimensional periodic potentials in terms of a matrix continued fraction expansion ${ }^{10}$ has been used by Ferrando et al. ${ }^{11,12}$ to discuss different diffusion regimes as well as to test the predictions of Kramers theory about escape rates. Activated rate processes in one-dimensional surface diffusion have been studied by Pollak et al. ${ }^{13}$ Furthermore, multiple hops in bidimensional activated surface diffusion have been recently observed ${ }^{14}$ in the surface diffusion of $\mathrm{Pt}$ on $\operatorname{Pt}(110)$ and theoretically interpreted by Pollak and coworkers. ${ }^{15}$ An alternative way to calculate such rates is provided by the well known concept of mean first passage time

$\dagger$ Presented at the Second International Meeting on Photodynamics, Havana, Cuba, February 10-16, 2002.
(MFPT), $\tau_{\mathrm{MFPT}}$, from the theory of stochastic processes. By this time we mean the average time elapsed until a stochastic process starting out at a given point leaves a prescribed domain for the first time. ${ }^{10}$ The escape rate is simply determined by the inverse of the MFPT. For large barriers, MFPT calculations are very inefficient. This relation between the MFPT and the escape rate, as calculated in Kramers work by the total flux of particles over the initial population of the well, has been demonstrated for the special case of a Gaussian white noise in Appendix B of ref. 1, and only very recently has been shown to rigorously hold for arbitrary time-homogeneous stochastic processes. ${ }^{16}$ Again, starting usually with a Fokker-Planck type equation, working analytical formulas can be obtained for simple interaction potentials for one, ${ }^{1}$ several dimensions ${ }^{17}$ in the weak noise limit and for two types of transport regimes, normal ${ }^{1}$ and anomalous. ${ }^{18,19}$ A second theoretical approach in transport phenomena consists of starting with a many-body Liouville equation, and applying projection operator formalisms. ${ }^{20,21}$ Then a (generalized) Langevin-type equation is recovered for the relevant slow variables. These projection techniques as well as the memory function have been shown to also give suitable theoretical support for a successful description of a wide variety of relaxation processes. ${ }^{22,3}$ Obviously, both kinds of theoretical approaches are equivalent.

In surface adatom diffusion, a lot of experimental information can be found in the literature spurring a major theoretical interest in interpreting the huge number of data at our disposal. $^{5}$ As far as we know, the first experimental observation of jump diffusion of isolated atoms on metal surfaces by quasielastic helium atom scattering (QHAS) is due to Ellis et al. ${ }^{23}$ in particular, $\mathrm{Na}$ atoms on a $\mathrm{Cu}(001)$ surface. In ulterior works, new and extended experimental results have been published. ${ }^{24-26}$ On this system, Chen and Ying have also carried out molecular dynamics simulations based on the projection operator of Mori and Zwanzig, Langevin and Fokker-Planck formalisms and found good agreement with experiment. ${ }^{27-29}$ At the same time, several semiempirical potential energy surfaces (PES) have been proposed by fitting the reported experimental data. ${ }^{23,24,26}$ Very recently, we have carried out a detailed investigation of the classical regular and chaotic motion of isolated $\mathrm{Na}$ adatoms on a two-dimensional periodic potential surface simulating the $\mathrm{Cu}(001)$ surface. ${ }^{30}$ 
On the other hand, the Kramers model is specially well suited for this problem and its predictions can be experimentally tested since a Fokker-Planck, or equivalently a Langevin approach, with constant friction is found accurate enough under fairly general experimental conditions..$^{23,27,31,32}$ In our physical system, vibrational frequencies of the adsorbates, friction constants as well as noise assisted jump rates using approximate instantaneous jump models ${ }^{33,34,11}$ were obtained by comparison of the numerically calculated dynamic structure factor to the width of the quasielastic peak, which is assumed to have a Lorentzian shape, previous convolution with the apparatus response function. Prefactors for the activated jump rates were compared only to those given by ordinary TST, and the existence of multiple jumps was also mentioned, but only in the context of the instantaneous jump approximation (Chudley-Elliot model). ${ }^{25,27}$ Therefore, in our opinion, previous theoretical work has not been completed and further theoretical studies under alternative approaches are still lacking in the literature.

In the present paper, and using as a starting point the adiabatic interaction potentials provided by the experimental works, we want to test the predictions of the Kramers model for diffusion of $\mathrm{Na}$ atoms on a $\mathrm{Cu}$ surface, as well as the feasibility of numerical implementations for obtaining escape or jump rates based on the solution of a Langevin equation with ohmic friction. Specifically, rates will be calculated in the whole friction range by counting jumps using an energy loss criterion, as well as from first passage time distributions and the results will be compared to the analytical predictions of Kramers and Mel'nikov ${ }^{8}$ for a bimetastable potential as in ref. 11. Jump length distributions and diffusion coefficients are also obtained for the corresponding separable and nonseparable potentials. Our results are compared to those previously reported using jump diffusion models and the main differences are discussed.

In the next section, we briefly describe the adiabatic interaction potentials used in our calculations and the basic formalism employed to obtain quantities of experimental relevance such as rates, diffusion coefficients and dynamic structure factors. Next, we present the numerical approach and the analytical models used for comparison. We finish with a discussion of the results and conclusions regarding previous findings.

\section{II. $\mathrm{Na} / \mathrm{Cu}(001)$ system and basic formalism}

\section{A. Model potential}

At low coverages of $\mathrm{Na}$ atoms, like in many experimental conditions, ${ }^{23-25}$ the adsorbate-adsorbate interaction is disregarded and only adsorbate-substrate interaction is taken into account, being governed by a two-dimensional periodic PES. The perpendicular degree of freedom can be safely neglected to interpret experimental results for this system, since the frequency of vibration along this coordinate is much faster than parallel vibrations, and the corresponding surface is considered as being averaged over the $\mathrm{Na}$ vibrations normal to the surface. In theoretical simulations of QHAS experiments, two PES have been proposed. ${ }^{23,24}$ The most recent one is non-separable and was fitted to an expression of the form ${ }^{24,25}$

$$
V(x, y)=V_{0}(x, y)+V_{1}(x, y)+V_{2}(x, y) .
$$

The first term is a simple separable cosine potential

$$
V_{0}(x, y)=V_{0}[2-\cos (2 \pi x / a)-\cos (2 \pi y / a)]
$$

where $a$ is the lattice constant of the $\mathrm{Cu}(001)$ surface ( $a=2.557 \AA$ ), and $V_{0}=41.4 \mathrm{meV}$. The second term is added to produce a lowering of the potential barrier in the diagonal direction.

$$
\begin{aligned}
V_{1}(x, y)= & -A \sum_{m, n} \exp \left(-b\left\{\left[x / a-\left(m+\frac{1}{2}\right)\right]^{2}\right.\right. \\
& \left.\left.+\left[y / a-\left(n+\frac{1}{2}\right)\right]^{2}\right\}\right) .
\end{aligned}
$$

with $A=2 V_{0}$ and $b=11.8$. The addition of this term was necessary in order to explain the experimental observation of a high rate of diffusion paths along the diagonal directions. ${ }^{25}$ Finally, the third term is a non-separable part which changes the curvature near the minima and varies the difference between the potential at the minima and the bridge positions,

$$
\begin{aligned}
V_{2}(x, y)= & C V_{0} \pi^{2} \sum_{m, n}\left[(x / a-m)^{2}+(y / a-n)^{2}\right] \\
& \times \exp \left[-(x / a-m)^{2}-(y / a-n)^{2}\right]
\end{aligned}
$$

with $C=-0.2$. Note that for a periodic potential, the sums in eqn. (2.3) and eqn. (2.4) must run over the entire set of integer pairs $(m, n)$. In practice, for the Langevin simulations, we reduce the dynamics to a single Wigner-Seitz cell by imposing periodic boundary conditions and the sum over Gaussians is truncated up to some few tems (typically $m$ and $n$ vary between -10 and 10). The $x$ and $y$ directions are taken along the azimuths with Miller indices [110] and [110] respectively. The energy zero is taken at the minima $(x, y)=(0,0)$ of the potential wells, corresponding to hollow sites. The saddle point along the $x$ or $y$ directions, $(x, y)=( \pm a / 2,0)$ or $(x, y)=$ $(0, \pm a / 2)$, respectively, is at $74.64 \mathrm{meV}$, and the saddle point along the diagonal [100] or [010] azimuths is at $84.49 \mathrm{meV}$. There appear also small minima on the potential truncated hills corresponding to on-top sites above the copper atoms which are at energy $82.74 \mathrm{meV}$. We have recently analyzed in detail the classical dynamics in this PES ${ }^{30}$ and found that the on-top minima play an important role in stabilizing motions along diagonal directions, and could provide an explanation of the anomalously high rate of jumps along the [100] direction detected in the experiment. The maxima at the top hills are located at $85.51 \mathrm{meV}$. In the first theoretical simulations of QHAS experiments for this particular system, ${ }^{23}$ a separable potential consisting of only the $V_{0}(x, y)$ term, eqn. (2.2), was employed, with a saddle point slightly lower $(67 \mathrm{meV})$.

In the present work, we will use both interaction models (separable and non-separable) to calculate diffusion rates and jump distributions in order to reveal the distinctive features emerging from the potential energy coupling. From a purely Hamiltonian dynamics point of view, corresponding to the limit of low temperature and friction, the difference between the separable and coupled cases is very important, since a separable potential gives an integrable Hamiltonian and the classical dynamics consists of only stable orbits, below and above the saddle-point energy. Moreover, above the saddle point energy, trajectories along the $x$ or $y$ directions propagate in a ballistic way, diffusing freely, and no change of direction or trapping into a well occur. On the contrary, the non separable potential gives rise to instabilities and chaos. Trajectories with energies above the diffusion barrier can get trapped for long times in a well, due to the existence of stable rotating orbits not present in the integrable case. ${ }^{30}$ Transport properties in analogy to the noisy case can be defined ${ }^{35}$ (although the source of randomness is intrinsic here, due only to the classical dynamics) and, depending on the energy, two different transport regimes, normal and enhanced, can arise. ${ }^{30,36}$ At very low friction and temperature values features of the classical dynamics can still survive. ${ }^{30}$ As we increase the temperature, noise destroys most of them but it also induces new effects. Nevertheless, different behavior of the diffusion coefficient as a function of friction has been found for separable and nonseparable two dimensional potentials, ${ }^{12,37}$ therefore the coupling in the adiabatic interaction produces qualitative changes which are worth exploring. 


\section{B. General theory}

The dynamical information of interest is contained in the socalled time-dependent pair correlation function, or van Hove, or $G$ function. At low adatoms concentration, the $G$ function is only described by the so-called "self part", $G(\boldsymbol{R}, t)$, and accounts for the ensemble averaged probability density of finding an atom at the surface position $\boldsymbol{R}=(x, y)$ at time $t$, given that the same adatom was at the origin at some arbritary time $t=0$. Such a function obeys a Fokker-Planck equation for a periodic potential under the influence of temperature and friction, a Klein-Kramers type equation. Equivalently, the dynamics of individual adatoms considered as Brownian particles is also given by a Langevin equation written as

$$
\ddot{\boldsymbol{R}}=-\frac{1}{m} \nabla_{\boldsymbol{R}} V(\boldsymbol{R})-\gamma \dot{\boldsymbol{R}}+\frac{1}{m} \boldsymbol{F}_{\mathrm{r}}(t)
$$

where $V$ is the adiabatic adsorption potential, eqn. (2.1), $\gamma$ the friction coefficient and $\boldsymbol{F}_{\mathrm{r}}$ a Gaussian white noise, that is

$$
\left\langle\boldsymbol{F}_{\mathrm{r}}(t) \boldsymbol{F}_{\mathrm{r}}\left(t^{\prime}\right)\right\rangle=2 m \gamma k T \delta\left(t-t^{\prime}\right) .
$$

By solving numerically the Langevin equation, one can obtain the individual Brownian trajectories for the adparticles. The relevant correlation functions are calculated by averaging over stochastic paths and time. For instance, the van Hove function $G(\boldsymbol{R}, t)$ is directly related to what is measured experimentally (the differential reflection probability for a projectile helium atom to be scattered into a certain solid angle $\Omega$ with an energy exchange $\hbar \omega$ ) through ${ }^{23,25}$

$$
\frac{\mathrm{d}^{2} R(\Delta \boldsymbol{K}, \omega)}{\mathrm{d} \Omega \mathrm{d} \omega}=n_{\mathrm{d}} F^{2} \iint G(\boldsymbol{R}, t) \mathrm{e}^{\mathrm{i}(\Delta \boldsymbol{K} \cdot \boldsymbol{R}-\omega t)} \mathrm{d} \boldsymbol{R} \mathrm{d} t
$$

with $\Delta \boldsymbol{K}$ being the wave vector transfer parallel to the surface, $n_{\mathrm{d}}$ the diffusing particle concentration on the surface and $F$ the atomic form factor depending on the interaction potential between the He atoms and the adparticles. The double integral in the right hand side of eqn. (2.7) is the dynamic structure factor, $S(\Delta K, \omega)$. Such a factor can also be expressed $a^{22}$

$$
S(\Delta \boldsymbol{K}, \omega)=\int \mathrm{d} t \mathrm{e}^{-\mathrm{i} \omega t}\left\langle\mathrm{e}^{-\mathrm{i} \Delta \boldsymbol{K} \cdot \boldsymbol{R}(t)} \mathrm{e}^{\mathrm{i} \Delta \boldsymbol{K} \cdot \boldsymbol{R}(0)}\right\rangle
$$

where the bracket in the integral means an ensemble average, and the positions $\boldsymbol{R}(t)$ can be obtained directly from the numerical solution of the Langevin eqn. (2.5) with initial condition $\boldsymbol{R}(0)$.

In order to extract information about jump rates or diffusion coefficients from the dynamic strucure factor, one makes approximations to the true Klein-Kramers dynamics valid in certain limits. As it is well known from the properties of the Fourier transform, scattering at small $\Delta \boldsymbol{K}$ provides information on long distance correlations. Therefore at very low $\Delta \boldsymbol{K}$ values the effect of the interaction potential is neglected (continuous diffusion model) and the van Hove function is assumed to obey a diffusion equation, whose solution is Gaussian, ${ }^{38}$ so that the dynamic structure factor becomes a Lorentzian function,

$$
S(\Delta \boldsymbol{K}, \omega)=\frac{1}{\pi} \frac{D \Delta \boldsymbol{K}^{2}}{\omega^{2}+D^{2} \Delta \boldsymbol{K}^{4}} .
$$

The diffusion coefficient $D$ can then be extracted from the full width at half maximum (FWHM) of the quasielastic peak, $\Gamma=2 D\left|\Delta \boldsymbol{K}^{2}\right|$. On the other hand, for activated processes $\left(E_{\mathrm{b}} \gg k T\right.$, where $E_{\mathrm{b}}$ is the energetic barrier for diffusion), residence times of the adatoms inside the potential wells are much longer than velocity correlation times, $\tau_{v} \sim 1 / \gamma$, and jumps between different sites are supposed to be instantaneous. Therefore, the Fokker-Planck dynamics can be approximated by a discrete master equation which can be solved by Fourier transformation. ${ }^{33,34}$ In this case the dynamic structure factor is again a Lorentzian, and the FWHM is related to the total jump rate and jump probabilities as

$$
\Gamma(\Delta \boldsymbol{K})=2 \kappa \sum_{j} P_{j}[1-\cos (\boldsymbol{j} \cdot \Delta \boldsymbol{K})]
$$

with $\kappa$ being the total jump rate out of an adsorption site and $P_{j}$ the relative probability for a jump with a displacement vector $\boldsymbol{j}$. We also note that for an isotropic adiabatic potential as in our case, the total jump rate and the diffusion coefficient are related by ${ }^{38,34}$

$$
D=\frac{1}{2 d} \kappa\left\langle\dot{j}^{2}\right\rangle
$$

where $d$ is the number of dimensions and $\left\langle\boldsymbol{j}^{2}\right\rangle$ the mean squared jump length (with value $a^{2}$ if only single jumps are considered). In order to apply eqn. (2.9) or (2.10) directly to the dynamic structure factor obtained experimentally, the assumption is made that diffusional and vibrational motions of the adsorbates are not correlated.

Solving the Langevin equation, eqn. (2.5), directly by numerical methods has the advantage that the quantities of interest, such as diffusion coefficients or rates, can be obtained by averaging over stochastic paths without the need to make any ad hoc approximation about the van Hove function. The price one has to pay is that sometimes the ensembles for the averages to be converged are very large, making the direct approach much more time consuming than, for instance, a matrix continued fraction solution or a finite element solution of the corresponding Fokker-Planck dynamics. An additional important advantage of the Langevin approach is that there exist simple numerical algorithms very easy to implement with quite good accuracy ${ }^{39,40}$ and, more importantly, the corresponding individual Brownian trajectories can be followed offering a direct and intuitive picture of some physical aspects of the diffusion process. The numerical method of our choice here to solve eqn. (2.5) is a third order velocity Verlet method, ${ }^{39}$ which gives a very good performance even when compared to methods of superior accuracy,${ }^{40}$ such as fourth order Runge-Kutta. Care must be taken in the selection of the time step, which we have chosen it to conserve the energy of the classical trajectories when taking the zero friction and temperature limits.

From Brownian dynamics simulations, correlation functions of interest, apart from the van Hove function, are the mean squared displacements, giving the diffusion coefficient through the Einstein relation

$$
D=\lim _{t \rightarrow \infty} \frac{1}{4 t}\left\langle|\boldsymbol{R}(t)-\boldsymbol{R}(0)|^{2}\right\rangle,
$$

and the velocity correlation function, which yields the power spectrum

$$
Z(\omega)=\int_{-\infty}^{\infty}\langle\boldsymbol{u}(t) \boldsymbol{u}(0)\rangle \mathrm{e}^{-\mathrm{i} \omega t} \mathrm{~d} t
$$

Note that $Z(\omega)$ also gives $D$ in the limit of small frequencies,

$$
D=\frac{1}{2} Z(\omega=0) \text {. }
$$

The power spectrum can be related to the dynamic structure factor in the $\Delta \boldsymbol{K} \rightarrow 0 \operatorname{limit}^{22}$ as an alternative to eqn. (2.13) but, in the present paper, we shall be concerned only with the calculation of jump rates and diffusion coefficients.

To calculate jump or escape rates we have propagated ensembles of trajectories obeying the Langevin dynamics eqn. (2.5), with initial conditions sampled from a MaxwellBoltzmann distribution centered in one potential well. For each trajectory we determine the jumps between different sites and finally calculate the jump rate as the total number of jumps of the ensemble, divided by the total propagation time. To count jumps we have chosen an energy criterion, similar to 
the one employed in ref. 37: a jump starts when a particle leaves the domain of a unit cell (namely, any of the coordinates crosses the saddle-point value for a trajectory initially thermalized in a well) and finishes when its energy is smaller than $\sim 3 k T$ below the potential barrier in a different potential well, since it is assumed that at this moment a thermalization of the particle in a well depth is attained. This somewhat arbitrary value of energy loss is converged as compared with a lower value (say $\sim 2 k T$ ), for the range of surface temperatures analysed. The energy criterion is slightly changed depending on the damping strength regime. Other criteria for the termination of a jump can be employed, such as the change of sign of velocity or the radius of curvature criterion used by Sholl and Skodje. ${ }^{41}$ The velocity criterion is misleading when considering diffusion in two dimensions, specially at high values of potential coupling, and at low friction values. ${ }^{42}$ The curvature criterion gives good results for the deterministic limit, ${ }^{30,41}$ but we find that the energy loss is of more physical relevance, above all if we want to compare to Kramers theory where one fundamental assumption is thermalization in a potential well before escaping.

In order to assess the validity of the energy loss criterion we have also calculated the escape rate from a different numerical strategy, namely, by obtaining the mean first passage time of crossing a given boundary for the initially thermalized ensemble. Such a time is almost independent of the precise location of the exit point as long as it is located at a sufficiently remote distance from the barrier. ${ }^{1}$ For periodic potentials with two equivalent exit paths along each direction, the escape rate in one direction ( $\operatorname{say} x$ ) is simply related to the mean first passage time $\left(\tau_{\mathrm{MFPT}}\right)$ by

$$
\kappa=\frac{1}{2 \tau_{\mathrm{MFPT}}} .
$$

We have checked that both approaches give the same result within numerical accuracy. At high values of friction, the value of $3 k T$ for the energy loss criterion should be modified in order to achieve convergence and consistency with the mean first passage time value. One of the additional advantages of the MFPT procedure is its numerical simplicity since trajectories are propagated only until they cross the chosen boundary.

\section{Numerical results}

We have studied first the separable potential, eqn. (2.2), since this can be reduced to two independent 1D problems along each direction, and analytical results are available mainly for the one dimensional case. The Langevin equation has been solved for ensembles of 20000 trajectories, and rates are calculated by the method outlined above. The temperatures considered here, which are also relevant to experiments, are between 100 and $200 \mathrm{~K}$. This implies that the ratio $E_{\mathrm{b}} / k T$ lies between 8 and 4 and we are safely in the activated regime, where Kramers theory applies (it is usually seen that for $E_{\mathrm{b}} / k T<2$ the Arrhenius behavior is $\operatorname{lost}^{12}$ ). Within this theory, and assuming a one dimensional parabolic barrier, say along the $x$ direction, with height $E_{\mathrm{b}}$ and frequency $\omega_{\mathrm{b}}=\sqrt{V^{\prime \prime}\left(x_{\mathrm{b}}\right) / m}$, the escape rate of the diffusing particles can always be written in the form

$$
\kappa=\mu \kappa_{\mathrm{TST}}=\mu \frac{\omega_{0}}{\pi} \exp \left(-E_{\mathrm{b}} / k T\right)
$$

where $\kappa_{\mathrm{TST}}$ is the transition state theory result (with a factor 2 which accounts for the two symmetric paths of escape out of the potential well), which usually overestimates seriously the true rate, and $\mu$ is a transmission factor taking into account the effects of friction and recrossings of the barrier before the particle finally escapes, not considered in the standard TST. The transmission factor can be expressed as

$$
\mu=\mu_{\mathrm{sd}} A(\Delta)
$$

where $\mu_{\mathrm{sd}}$ is the original Kramers prefactor ${ }^{7}$ for the spatial diffusion regime (moderate to high frictional damping)

$$
\mu_{\mathrm{sd}}=\sqrt{1+\gamma^{2} / 4 \omega_{\mathrm{b}}^{2}}-\gamma / 2 \omega_{\mathrm{b}}
$$

and

$$
A(\Delta)=\exp \left[\frac{1}{\pi} \int_{0}^{\infty} \frac{\ln \left\{1-\exp \left[-\Delta\left(x^{2}+\frac{1}{4}\right)\right]\right\}}{x^{2}+\frac{1}{4}} \mathrm{~d} x\right]
$$

where $\Delta$ is the dimensionless energy loss per oscillation of a particle close to the top of the barrier. eqn. (3.16)-(3.19) were first derived by Mel'nikov and Meshkov ${ }^{43}$ for a particle in a single potential well, providing the first complete solution of the Kramers turnover problem bridging the energy and spatial diffusion regimes. Pollak and coworkers ${ }^{1,44}$ generalized the theory to arbitrary time-dependent friction and obtained a systematic solution of the turnover problem using a normal-mode approach, giving Mel'nikov's eqn. (3.16)-(3.19) again, the only difference being in the calculation of the energy loss parameter $\Delta$. Ferrando et al. ${ }^{11}$ applied such equations for the escape problem from a bimetastable potential when comparing to the jump-rate problem in a periodic potential. This is what we have also followed in this work. The energy loss is obtained to lowest order in the frictional damping and inverse barrier height, ${ }^{43}$ which for the cosine potential with amplitude $V_{0}$ gives

$$
\Delta=\frac{\gamma}{k T} \int_{-a / 2}^{a / 2} \sqrt{2 m\left[E_{\mathrm{b}}-V(x)\right]} \mathrm{d} x=\frac{8 V_{0} \gamma}{k T \omega_{\mathrm{b}}} .
$$

The Mel'nikov-Meshkov (MM) result, eqn. (3.16)-(3.20), is plotted in Fig. 1, with solid lines and at $110 \mathrm{~K}$, as a function of the scaled friction $\gamma / \omega_{0}$ with $\omega_{0}=2 \pi \sqrt{V_{0} / m a^{2}}$ being the frequency of oscillation near the well bottom. For comparison, the corresponding numerical rates are also displayed: with black triangles, the full numerical resolution of the Langevin equation with the above mentioned energy loss criterion; and, with open circles, the MFPT values. The small discrepancy found, around the turnover region and underdamped region, between the MM result and the full numerical one can be mainly attributed to two factors: first, the parabolic barrier approximation explicitly assumed around the saddle point and, second, multiple hops are neglected in this model since the periodicity in the potential is not taken into account. ${ }^{13}$ The MFPT result is obtained by using a dividing surface located at $0.2 \AA$ away from the cell boundary where no back-crossing jumps are observed.

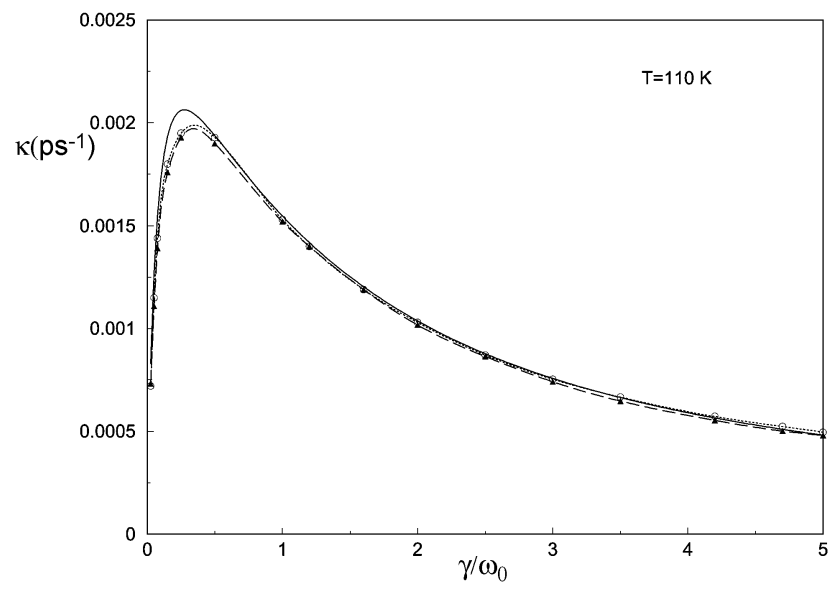

Fig. 1 Jump rates (in $\mathrm{ps}^{-1}$ ) versus the scaled friction $\gamma / \omega_{0}$ for the separable potential at $110 \mathrm{~K}$. Dotted-circle curve: MFPT values. Dashed-triangle curve: numerical results with the energy criterion. Solid curve: analytical MM jump rates, eqn. (3.16)-(3.19). 
For the Kramers model, MM gave an estimation of the probability of recrossings calculated as the backward-to-forward rate of fluxes of particles with opposite velocities ${ }^{43}$

$$
\frac{j^{\mathrm{B}}}{j^{\mathrm{F}}}=1-\left(1+\frac{\gamma^{2}}{4 \omega_{\mathrm{b}}^{2}}\right)^{-1 / 2}
$$

with $\mathrm{B}$ for backward and $\mathrm{F}$ for forward fluxes. In the underdamped regime, $\gamma \ll \omega_{\mathrm{b}}$, recrossings can be safely neglected, but in the moderate-to-high friction regime they can make an important contribution due to the influence of the noise. However, as reported by Pollak et $a l^{44}$ the recrossing problem does not occur in the normal mode representation of the problem. It has been shown that the best dividing surface may be considered as a collective mode which is a linear combination of the system coordinate and all bath modes.

The coupling in the interaction potential also influences the rate and diffusion coefficient. In Fig. 2, rates calculated by the MFPT method at $T=200 \mathrm{~K}$ for the potential without coupling (2.2) (dashed line) and with coupling, eqn. (2.1)-(2.4), (solid line) are shown. For comparison, in both calculations the barrier heights are the same so that the difference between both curves is only due to the coupling. In contrast to the findings of other authors, ${ }^{12}$ the rate is here increased in the non-separable case. We mention that in the potential studied by them, the coupling makes the channels at the saddle-points narrower, while for the $\mathrm{Na} / \mathrm{Cu}$ non-separable potential studied the effect is the opposite, thus making the crossing at the dividing surface easier. For the diffusion coefficient, not only the total jump rate but also mean squared jump lengths are important (see eqn. (2.11)). At low values of friction, it has been found that multiple jumps have appreciable probabilities. $^{11,15,25,28}$ Keeping the temperature not too high, so that we are in the activated regime, one expects that increasing the temperature will also enhance the probability of multiple jumps. In Fig. 3 the combined effect of temperature and potential coupling on the jump length distribution as a function of unit cell length number is plotted. Jump distributions at two different temperatures are shown, $T=110 \mathrm{~K}$ (circles) and $T=200 \mathrm{~K}$ (triangles) for the separable (solid lines) and nonseparable (dashed line) interaction potentials, at $\gamma=0.1 \omega_{0}$, the experimental value obtained for friction. The fraction of double and triple jumps in the non-separable potential is $13 \%$ and $4 \%$ at $110 \mathrm{~K}$, and $14 \%$ and $8 \%$ at $200 \mathrm{~K}$, respectively. As expected, not only long jumps are favored by increasing the temperature, but also the coupling plays a role, leading to an enhancement of the multiple-jump probabilities and the

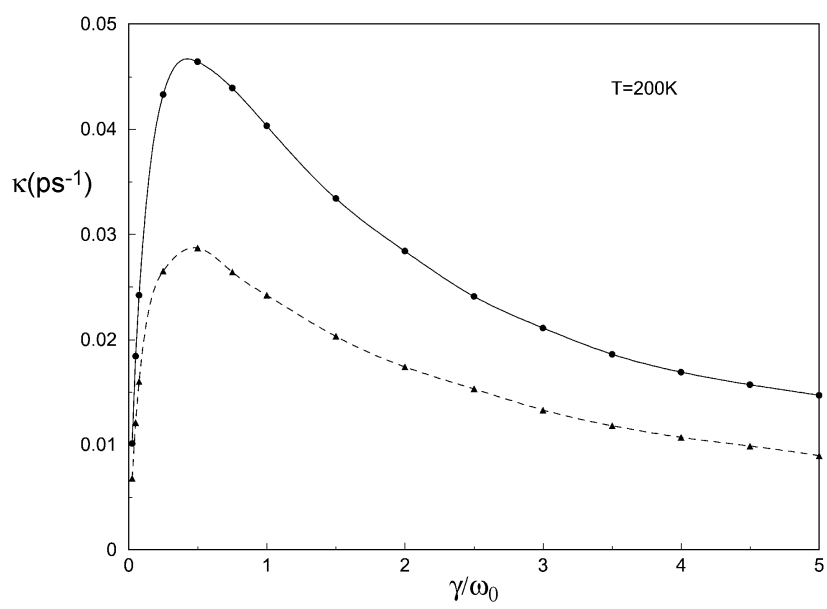

Fig. 2 Numerical MFPT jump rates (in $\mathrm{ps}^{-1}$ ) versus the scaled friction at $200 \mathrm{~K}$. Solid curve: nonseparable periodic potential. Dashed curve: separable periodic potential. For comparison, the diffusion barrier is in both calculations the same, $74.64 \mathrm{meV}$.

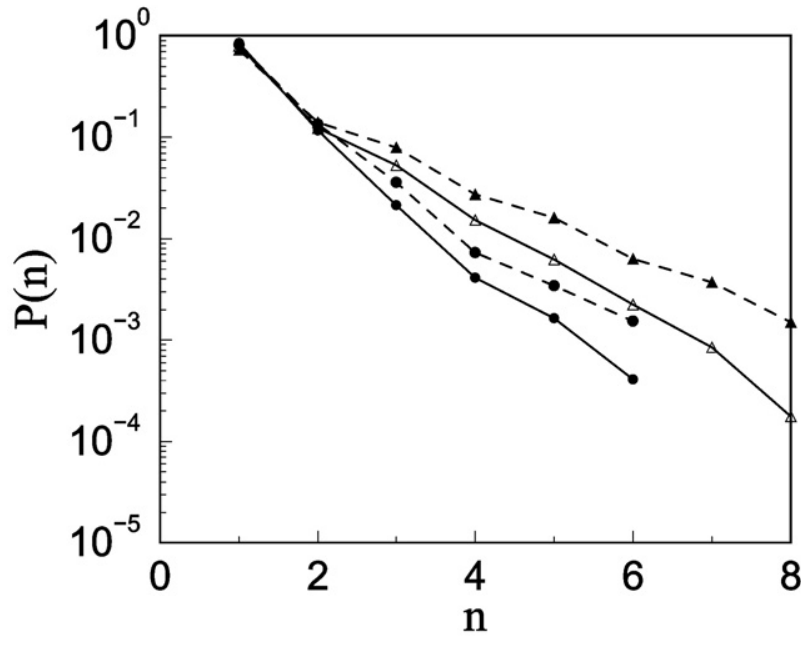

Fig. 3 Jump probabilities versus jump distance (in units of lattice constant) for the separable (solid lines) and nonseparable (dashed lines) periodic potentials at two different temperatures. Circles, 110 $\mathrm{K}$; Triangles, $200 \mathrm{~K}$.

diffusion coefficient. Diffusion coefficients depend on the jump rate through eqn. (2.11) and, on the other hand, they can be also numerically obtained using the Einstein relation eqn. (2.12) at sufficiently long times. In Fig. 4, at $200 \mathrm{~K}$ and for the separable (squares) and nonseparable (circles) periodic potentials, it is plotted the diffusion coefficient as a function of the dimensionless friction calculated by the Einstein relation (2.12) (solid curves) and using eqn. (2.11) with the rate obtained by counting jumps with the energy criterion (dotted curves) observing very good agreement in each case. As expected by the previous calculations, the diffusion coefficients are higher for the nonseparable potential.

\section{Conclusions and comparison to experimental data}

Quasielastic peaks are always centered at $\Delta E=\hbar \omega=0$ and information of the diffusional motion of the adsorbate can be only extracted from their broadenings. Effective Lorentzian line shapes are used to convolute the experimental quasielastic peaks and obtain information on the friction coefficient,

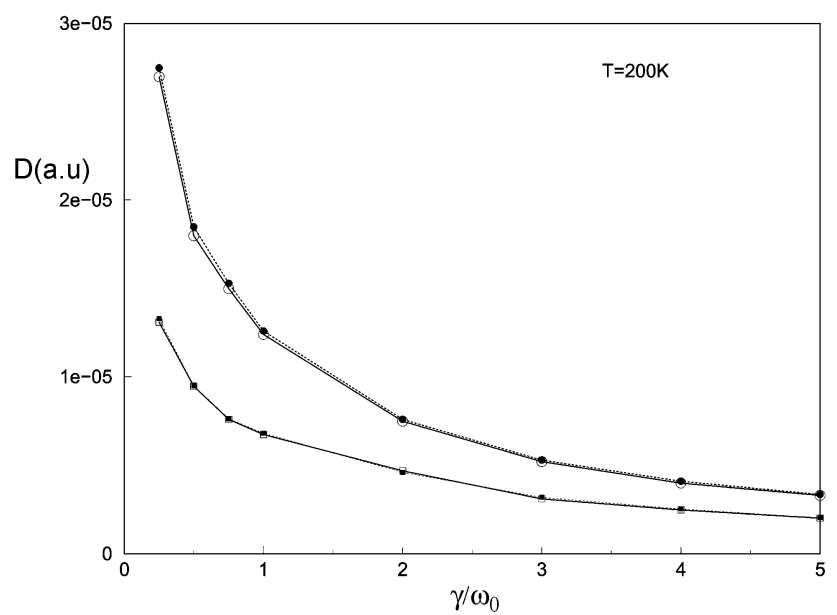

Fig. 4 Diffusion coefficient in atomic units as a function of the scaled friction at $200 \mathrm{~K}$ for the separable (black and white squares) and nonseparable (black and white circles) periodic potentials. Solid curves: Einstein relation, eqn. (2.12). Dotted curves: Eqn. (2.11) using the energy criterion (see text) for the jump rate. 
vibrational frequencies and total jump rates. ${ }^{23-25}$ In order to obtain diffusion coefficients and jump rates the Chudley-Elliot model has been widely assumed. ${ }^{33}$ Furthermore, Arrhenius plots obtained from best fits to experimental results compared to theoretical (molecular dynamics) calculations and TST behavior are the main ingredients used in interpreting atomsurface diffusion experiments. For the $\mathrm{Na} / \mathrm{Cu}(001)$ system, the friction reported in the literature is $0.1 \omega_{0}$ and, therefore, we are in the turnover region of the Kramers model but closer to the low friction (energy diffusion) regime (see Fig. 1).

The separable cosine potential used in the first simulations of the experiments for this system ${ }^{23,27}$ was found to overestimate the barrier for diffusion along diagonal ([100]) azimuths, therefore the full non-separable potential was proposed to account well for all the experimental findings. Chen and Ying, ${ }^{27}$ using the Mori projection formalism and the instantaneous jump model, calculated the jump rates in the separable potential, for a range of temperature between 200-300 K and found an activated behavior with a prefactor $\mu \sim 0.1$ in eqn. (3.16) (note that they use the expression $\kappa_{\mathrm{TST}}=\left(\omega_{0} / 2 \pi\right)$ $\exp \left(-E_{\mathrm{b}} / k T\right)$ for the TST rate, while the factor $1 / 2$ should be removed for the periodic potential). We have found an activated Arrhenius behavior (Fig. 5(a)) of the jump rates for the same temperature range, using both the MFPT procedure and the energy criterion for counting jumps with a prefactor $\mu \sim 0.22$. The corresponding calculations have been carried out for a friction value of $0.15 \omega_{0}$ with $\omega_{0}=9.31 \mathrm{THz}$. The effective diffusion barrier calculated from the Arrhenius fitting is around $52 \mathrm{meV}$, which agrees well with the value of $E_{\mathrm{b}}=51 \pm 6 \mathrm{meV}$ obtained experimentally. ${ }^{23}$
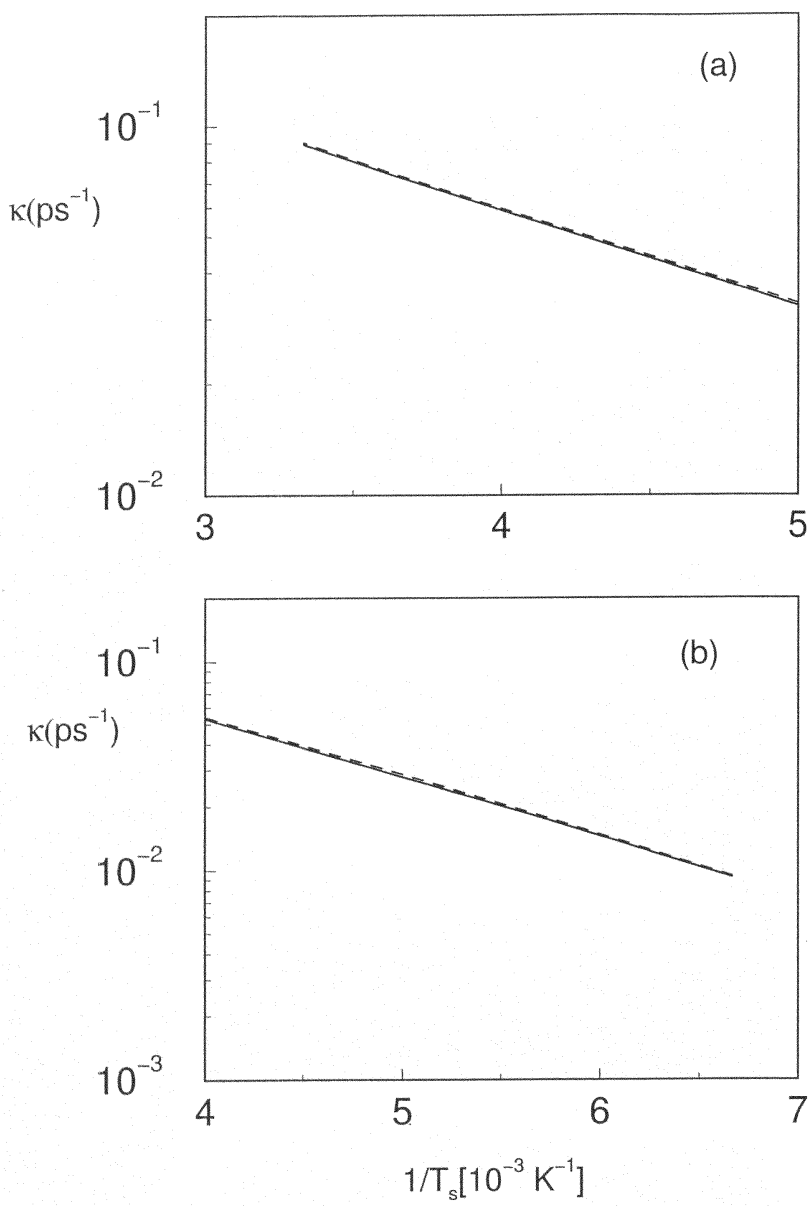

Fig. 5 Arrhenius plots for the (a) separable and (b) nonseparable periodic potentials. Solid lines are the MFPT results and dashed lines reports the numerical results wih the energy criterion (see text).
For the non-separable potential, Arrhenius prefactors were directly extracted from a fitting of the experimental quasielastic peak to the Lorentzian form, eqn. (2.9), at small $\Delta \boldsymbol{K}$ values and different temperatures (between 150-250 K), giving the result $\mu=3.3 \pm 0.3^{25}$ versus the TST result of $\mu=1$. This result is quite surprising because we have to remember that the TST usually overestimates the true rate. Furthermore, in Fig. 5(b), we plot our numerical results calculated at a friction value of $0.1 \omega_{0}$ with $\omega_{0}=9 \mathrm{THz}$ and the corresponding prefactor is $\mu \sim 0.23$ and the effective barrier $E_{\mathrm{b}} \sim 55 \mathrm{meV}$. In our opinion, the reason of this strong discrepancy is that those authors obtain the prefactor from the diffusion coefficient given as (see eqn. (2.11)) $D=0.5 \mu \omega_{0} a^{2} / \pi$, while at these low values of friction the contribution of multiple jumps are important and mean squared jump lengths are greater than $a^{2}$. For instance, they reported a fraction of $20 \%$ of double jumps and around $5 \%$ of triple jumps. ${ }^{25}$

In conclusion, in this work, the influence of the potential coupling has been investigated and shown to have an effect in jump rates, jump length probabilities, and diffusion coefficients, in the whole damping range, due mainly to the particular shape of the potential around the saddle-point barrier. Discrepancies with respect to previous experimental findings in the $\mathrm{Na} / \mathrm{Cu}(001)$ system have been also reported. Finally, due to the fact that the agreement found between our numerical calculations and the extended Kramers theory is fairly good, such a theory should be widely used in activated surface diffusion problems instead of the standard transition state theory.

\section{Acknowledgements}

This work has been supported in part by DGICYT (Spain) under contract BFM2001-2179. J. L. V. and R. G. would like to thank the Minister of Science and Technology (Spain) for a predoctoral grant and a Ramón y Cajal Contract. We would like to thank Prof. E. Pollak for his very interesting suggestions and comments.

\section{References}

1 P. Hänggi, P. Talkner and M. Borkovec, Rev. Mod. Phys., 1990, 62, 251 .

2 T. Fonseca, J. A. N. F. Gomes, P. Grigolini and F. Marchesoni, Adv. Chem. Phys., 1985, 62, 389.

3 E. Pollak, in Dynamics of Molecules and Chemical Reactions, ed. R. E. Wyatt and J. Z. H. Zhang, Marcel Dekker Inc., New York, 1996, ch. 16 , p. 617

4 R. Gomer, Rep. Prog. Phys., 1990, 53, 917.

5 J. V. Barth, Surf. Sci. Rep., 2000, 40, 75.

6 J. A. Christiansen, Z. Phys. Chem. B, 1936, 33, 145.

7 H. Kramers, Physica, 1940, 7, 284.

8 V. I. Mel'nikov, Phys. Rep., 1991, 209, 1.

9 W. T. Coffey, D. A. Garanin and D. J. McCarthy, Adv. Chem. Phys., 2001, 117, 483.

10 H. Risken, The Fokker-Planck Equation, 1989, Springer, Berlin.

11 R. Ferrando, R. Spadacini and G. E. Tommei, Phys. Rev. A, 1992, 46, R699; R. Ferrando, R. Spadacini and G. E. Tommei, Phys. Rev. E, 1993, 48, 2437.

12 G. Caratti, R. Ferrando, R. Spadacini and G. E. Tommei, Phys. Rev. E, 1996, 54, 4708; G. Caratti, R. Ferrando, R. Spadacini and G. E. Tommei, Chem. Phys., 1998, 235, 157.

13 J. S. Bader, B. J. Berne and E. Pollak, J. Chem. Phys., 1995, 102 4037; Y. Georgievskii, M. A. Kozhushner and E. Pollak, J. Chem. Phys., 1995, 102, 6908.

14 T. R. Linderoth, S. Horch, E. Laegsgaard, I. Stensgaard and F. Besenbacher, Phys. Rev. Lett., 1997, 78, 4978.

15 E. Hershkovitz, P. Talkner, E. Pollak and Y. Georgievskii, Surf. Sci., 1999, 421, 73.

16 P. Reimann, G. J. Schmid and P. Hänggi, Phys. Rev. E, 1999, 60, R1.

17 P. Talkner, Z. Phys. B, 1987, 68, 201. 
18 G. Rangarajan and M. Ding, Phys. Rev. E, 2000, 62, 120; M. Gitterman, Phys. Rev. E, 2000, 62, 6065.

19 R. Metzler and J. Klafter, Adv. Chem. Phys., 2001, 116, 223.

20 R. Zwanzig, J. Chem. Phys., 1960, 33, 1338.

21 H. Mori, Prog. Theor. Phys., 1965, 33, 423; H. Mori, Prog. Theor. Phys., 1965, 34, 399.

22 J. P. Hansen and I. R. McDonald, Theory of Simple Liquids, Academic Press, London, 1986.

23 J. Ellis and J. P. Toennies, Phys. Rev. Lett., 1993, 70, 2118; A. P. Graham and J. P. Toennies, Surf. Sci., 1999, 427-428, 1.

24 A. P. Graham, F. Hofman, J. P. Toennies, L. Y. Chen and S. C. Ying, Phys. Rev. Lett., 1997, 78, 3900.

25 J. W. M. Frenken and B. J. Hinch, in Helium Atom Scattering from Surfaces, ed. E. Hulpke, Springer Series in Surface Science vol. 27, Springer-Verlag, New York, 1992, p. 287; A. P. Graham, F. Hofman and J. P. Toennies, L. Y. Chen, S. C. Ying, Phys. Rev. $B, 1997, \mathbf{5 6}, 10567$.

26 J. Ellis, A. P. Graham, F. Hofman and J. P. Toennies, Phys. Rev. $B, 2001,63,195408$.

27 L. Y. Chen and S. C. Ying, Phys. Rev. Lett., 1993, 71, 4361

28 L. Y. Chen and S. C. Ying, Phys. Rev. B, 1994, 49, 13838.

29 A. Cucchetti and S. C. Ying, Phys. Rev. B, 1999, 60, 11110.

30 R. Guantes, J. L. Vega and S. Miret-Artés, Phys. Rev. B, 2001, 64, 245415; J. L. Vega, R. Guantes and S. Miret-Artés, J. Phys.: Condens. Matter, 2002, 14, 6193
31 D. C. Senft and G. Ehrlich, Phys. Rev. Lett., 1995, 74, 294

32 Y. Georgievskii and E. Pollak, Surf. Sci., 1996, 355, L366.

33 C. T. Chudley and R. J. Elliot, Proc. Phys. Soc. London, 1961, 77, 353.

34 G. Wanshtröm, in Interactions of Atoms and Molecules with Solid Surfaces, ed. V. Bortolani, N. H. March and N. P. Tosi, Plenum Press, New York, 1990, p 529.

35 P. Gaspard, Chaos, Scattering and Statistical Mechanics, Cambridge University Press, 1998, Nonlinear Science Series IX.

36 T. Geisel, A. Zacherl and G. Radons, Phys. Rev. Lett., 1987, 59, 2503.

37 L. Y. Chen, M. R. Baldan and S. C. Ying, Phys. Rev. B, 1996, 54, 8856.

38 S. Chandrasekhar, Rev. Mod. Phys., 1943, 15, 1.

39 M. P. Allen and D. J. Tildesley, Computer Simulations of Liquids, Clarendon Press, Oxford, 1987.

40 E. Hershkovitz, J. Chem. Phys., 1998, 108, 9253; See also R. L. Honeycutt, Phys. Rev. A, 1992, 45, 600.

41 D. S. Sholl and R. T. Skodje, Physica D, 1994, 71, 168

42 R. Ferrando, R. Spadacini and G. E. Tommei, Phys. Rev. E, 1995 51, 126.

43 V. I. Mel'nikov and S. V. Meshkov, J. Chem. Phys., 1986, 85, 1018.

44 E. Pollak, H. Grabert and P. Hänggi, J. Chem. Phys., 1989, 91, 4073 . 